\title{
EFFECTS OF PORTAL VENOUS INOCULATION WITH DONOR SPLENOCYTES ON LUNG ALLOGRAFT SURVIVAL IN DOGS
}

Hideki Ichinari, MD

Tetsuya Shimizu, MD, PhD

Makoto Yoshioka, MD, PhD

Yasunori Matsuzaki, MD, PhD

Koichiro Shibata, MD, PhD

Yasunori Koga, MD, PhD
The effects of portal venous inoculation with donor splenocytes on lung allograft survival were evaluated in dogs. Within the dose range examined, portal venous inoculation with donor splenocytes alone did not affect the graft survival time. Although the mean graft survival time was prolonged by FK 506 treatment for 14 days, the transplanted lungs were rejected within 12 days after termination of FK 506 administration. However, when the recipients were given portal venous inoculation with donor splenocytes at the time of transplantation, the graft survival time after termination of FK 506 administration was significantly prolongated. In the recipients that received combined therapy of portal venous inoculation with donor splenocytes and FK 506 treatment, arterial oxygen tension and histologic architecture of the transplanted lungs remained within the normal range for a longer period than these measures did in control animals that received FK 506 treatment alone. Dose-response experiments revealed that $1 \times 10^{8}$ cells/recipient was most effective in causing prolonged graft survival after termination of FK 506 treatment. On the basis of these results, perioperative portal venous inoculation with donor splenocytes can enhance the immunosuppressive effect of FK 506 in the canine lung transplantation model. (J Thorac Cardiovasc Surg 1996;112:300-5)
Qince lung transplantation was first attempted $\checkmark$ clinically in 1963 by Hardy and coworkers, ${ }^{1}$ it has been widely applied for the treatment of end-stage lung diseases. ${ }^{2,3}$ From the technical standpoint, lung transplantation has been established as a useful therapeutic modality. The most important problem yet to be solved is to control the host's immune responses against transplanted organs. Although immunosuppressants such as cyclosporine and FK 506 are used to overcome the host's rejection reaction, side effects of these drugs are clinically a major drawback. ${ }^{4,5}$

Therefore it is necessary to develop new therapeutic procedures to reduce or avoid the use of immunosuppressants. One such way is to induce immunologic tolerance against donor antigens. In

From the Second Department of Surgery, Miyazaki Medical College, Miyazaki, Japan.

Received for publication May 18, 1995; accepted for publication Oct. 3, 1995.

Address for reprints: Hideki Ichinari, MD, The Second Department of Surgery, Miyazaki Medical College, 5200 Kiwara, Kiyotake, Miyazaki 889-16, Japan.

Copyright (c) 1996 by Mosby-Year Book, Inc.

$0022-5223 / 96 \$ 5.00+0 \quad \mathbf{1 2 / 1 / 6 9 6 6 2}$ rodent models of heart, kidney, and liver transplantations, graft survival could be prolonged by portal venous inoculation with donor lymphocytes. ${ }^{6-11}$ In particular, in a rat liver transplantation model, portal venous inoculation with donor splenocytes resulted in permanent graft survival. ${ }^{11}$ In experimental models with larger and genetically heterogenous animals, however, the efficacy of portal venous inoculation with donor splenocytes on graft survival remains unclear except in the recent work on the prolonged survival of renal allografts by this treatment in dogs. ${ }^{12,13}$ In the present study, we examined the effects of portal venous inoculation with donor splenocytes on graft survival in a canine lung transplantation model.

\section{Material and methods}

Animals. Adult mongrel dogs (body weight 8.0 to 14.5 $\mathrm{kg}$ ) were used. The study followed the guidelines for animal experimentation issued by Miyazaki Medical College, which were based on the "Guide for the Care and Use of Laboratory Animals" prepared by the National Institutes of Health (NIH Publication No. 86-23, revised 1985).

Lung transplantation. All dogs were anesthetized with sodium pentobarbital $(25 \mathrm{mg} / \mathrm{kg})$ by intravenous injection after an intramuscular injection of $0.5 \mathrm{mg}$ atropine sulfate. 
Table I. Effects of portal venous inoculation with donor splenocytes on graft survival in lung transplantation in dogs

\begin{tabular}{lccc}
\hline $\begin{array}{c}\text { No. of } \\
\text { cells }\end{array}$ & $\begin{array}{c}\text { No. of } \\
\text { animals }\end{array}$ & $\begin{array}{c}\text { Graft survival } \\
\text { time (days) }\end{array}$ & Mean $\pm S D$ \\
\hline None & 6 & $4,5,7,8,9,10$ & $7.2 \pm 2.3$ \\
$1.0 \times 10^{6}$ & 4 & $7,7,8,8$ & $7.5 \pm 0.6^{*}$ \\
$1.0 \times 10^{8}$ & 4 & $7,8,8,9$ & $8.0 \pm 0.8^{*}$ \\
$1.0 \times 10^{10}$ & 4 & $6,6,7,7$ & $6.5 \pm 0.6^{*}$ \\
\hline
\end{tabular}

$S D$, Standard deviation.

*Not significantly different from control group given no cells.

They were then intubated (outer diameter $8.5 \mathrm{~mm}$ ) and the lungs ventilated with a tidal volume of $30 \mathrm{ml} / \mathrm{kg}$ at a rate of 15 breaths $/ \mathrm{min}$, with $5 \mathrm{~cm} \mathrm{H}_{2} \mathrm{O}$ positive endexpiratory pressure. Allotransplantation of the left lung was done according to the method of Veith and Richards. ${ }^{14}$ The left lung harvested from a size-matched donor was perfused with $500 \mathrm{ml}$ of cold $\left(4^{\circ} \mathrm{C}\right)$ saline solution containing heparin $(10 \mathrm{U} / \mathrm{ml})$ via the pulmonary artery at a pressure of $40 \mathrm{~cm} \mathrm{H}_{2} \mathrm{O}$ and was kept on ice slush until transplantation. Cold ischemic time was less than 30 minutes and warm ischemic time was less than 60 minutes. An inflatable cuff was placed around the proximal right pulmonary artery of the recipient, which allowed independent assessment of transplanted lung function. ${ }^{15}$

Portal venous inoculation with donor splenocytes. The spleen was excised from the donor immediately before lung transplantation. The spleen was minced and pressed gently through a stainless steel mesh into phosphatebuffered saline solution. The cell suspension was centrifuged at $1500 \mathrm{rpm}$ for 5 minutes and resuspended in $\mathrm{NH}_{4} \mathrm{Cl}$ solution to remove red blood cells. The cells were washed twice and the designated number of donor splenocytes $(1 x$ $10^{8} \mathrm{cell} \mathrm{s} / \mathrm{ml}$ ) was infused via the superior mesenteric vein of the recipients 30 minutes before lung transplantation. ${ }^{12}$

Immunosuppressive drug treatment. FK 506 (Fujisawa Pharmaceutical Co., Ltd., Osaka, Japan) was dissolved in saline solution and was injected intramuscularly into the thigh. The dose of FK 506 was $0.1 \mathrm{mg} / \mathrm{kg}$ per day and the period of administration was for 14 days starting from the day of lung transplantation (day 0 ). Plasma trough levels of FK 506 were measured by a double antibody enzymelinked immunosorbent assay method. ${ }^{16}$

Experimental designs. In the first experiment, three groups of dogs ( 4 animals each) received portal injection with $1 \times 10^{6}, 1 \times 10^{8}$, or $1 \times 10^{10}$ donor splenocytes immediately before lung transplantation. An additional group ( 6 dogs) was given no cells but received lung transplantation to serve as a control group. In the second experiment, dogs were divided into three groups (6 animals each). The first group (FK group) was treated with FK 506 for 14 days after lung transplantation, the second group (PV group) received portal venous inoculation with $1 \times 10^{8}$ donor splenocytes immediately before transplantation, and the third group (PV + FK group) received portal venous inoculation with donor splenocytes immediately before transplantation and then treatment with FK 506 for 14 days. Graft survival time of each group was compared with that of the control group in the first
Table II. Effects of combined therapy with portal venous inoculation with donor splenocytes and $F K$ 506 administration on grafted lung in dogs

\begin{tabular}{lccc}
\hline Group & $\begin{array}{c}\text { No. of } \\
\text { animals }\end{array}$ & $\begin{array}{c}\text { Graft survival } \\
\text { time (days) }\end{array}$ & Mean $\pm S D$ \\
\hline Control & 6 & $4,5,7,8,9,10$ & $7.2 \pm 2.3$ \\
FK & 6 & $18,20,21,22,23,25$ & $21.5 \pm 2.4$ \\
PV & 6 & $4,5,5,6,7,10$ & $6.2 \pm 2.1$ \\
PV + FK & 6 & $26,33,34,36,36,63$ & $38.0 \pm 12.8^{*}$ \\
\hline
\end{tabular}

Control group, which is the same as that in Table I, received no cells and received lung transplantation alone. FK group was treated with FK 506 $(0.1 \mathrm{mg} / \mathrm{kg}$ per day) for 14 days starting from day of lung transplantation. PV group received lung transplantation preceded by portal venous inoculation with $1 \times 10^{8}$ donor splenocytes. PV + FK group received lung transplantation and perioperative portal venous inoculation with donor splenocytes $\left(1 \times 10^{8}\right.$ cells) and followed by FK 506 treatment for 14 days. $S D$, Standard deviation.

" $p<0.05$ versus control group, FK group, and PV group.

experiment. In the third experiment, two groups of animals ( 4 animals each) were given $1 \times 10^{6}$ and $1 \times 10^{10}$ donor splenocytes immediately before lung transplantation, and then they were treated with FK 506 for 14 days. Results in this group were compared with those of the FK group and also with results in the PV + FK group in the second experiment.

Assessments of graft survival. Grafts were evaluated for rejection manifested by opacification of the transplanted lung on chest roentgenograms. Radiologic examinations were done every 3 days, and when rejection was suspected the examinations were done every day. For histopathologic assessment of rejection, tissue specimens were obtained by open lung biopsy every 7 days after transplantation and on the day of rejection determined by radiology. Radiologic opacification corresponded to grade 3 (moderate rejection) or 4 (severe rejection) of the histopathologic classification of the International Society for Heart Transplantation. ${ }^{17}$

Functional tests of the transplanted lung. A right pulmonary artery occlusion test was done on the day of transplantation and every 7 days thereafter to evaluate the function of the transplanted lungs. The animals were maintained on a respirator under general anesthesia at a tidal volume of $30 \mathrm{ml} / \mathrm{kg}$, a respiratory rate of 15 breaths/ min, $5 \mathrm{~cm} \mathrm{H} \mathrm{H}_{2} \mathrm{O}$ positive end-expiratory pressure, and an inspired oxygen fraction of 1.0; arterial blood gas values were measured 10 minutes after occlusion of the right pulmonary artery.

Statistical analysis. All values were expressed as mean plus or minus standard deviation. The paired differences between groups were determined by analysis of variance with the StatView 4.0 (Abacus Concepts, Inc., Berkeley, Calif.) software package. Differences were considered to be significant when $p$ values were less than 0.05 .

\section{Results}

In the first experiment, effects of portal venous inoculation with donor splenocytes alone on lung graft survival were examined. Three groups of dogs (4 animals each) received portal injection with $1 \times$ 


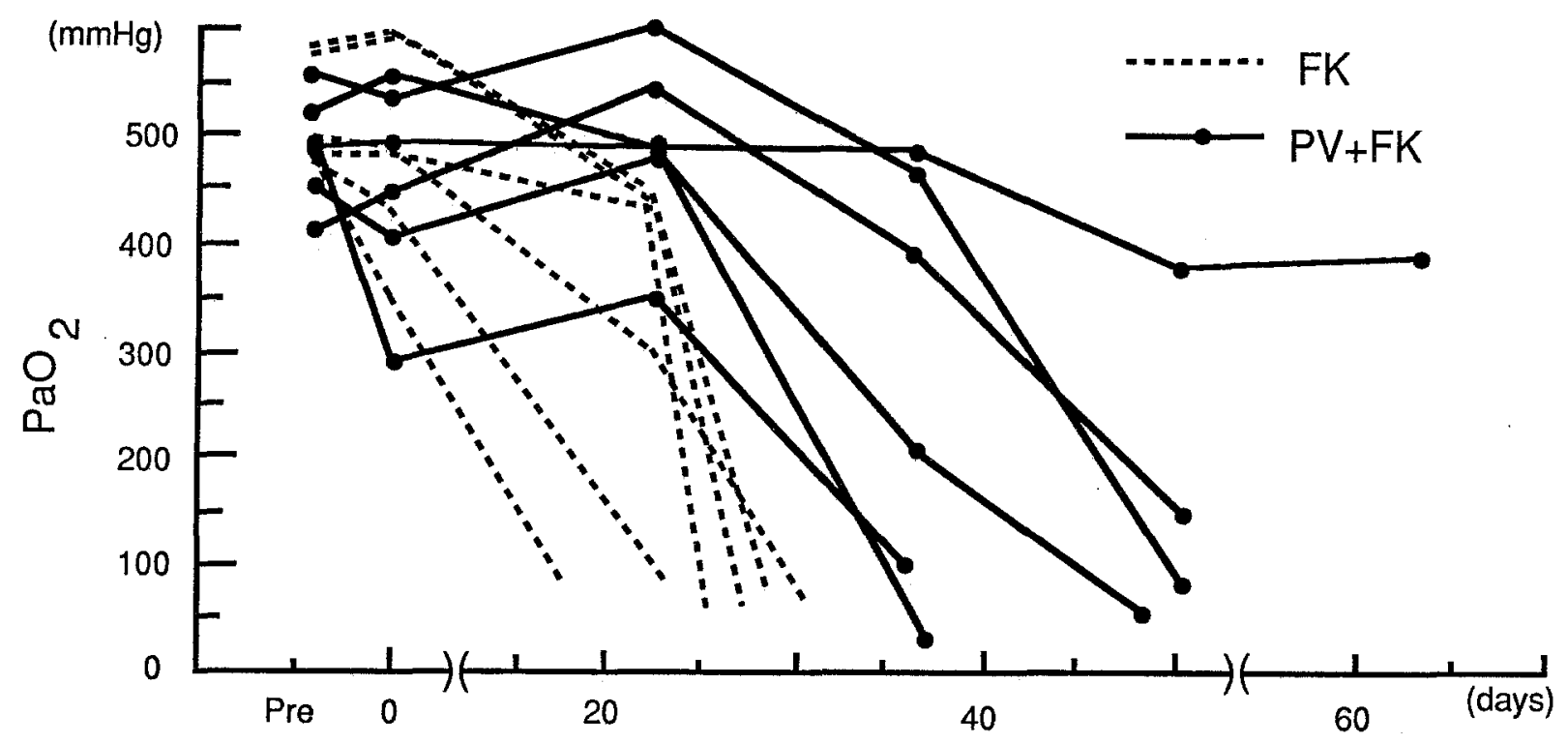

Days After Transplantation

Fig. 1. Functional test of transplanted lungs measured by arterial oxygen tension $\left(\mathrm{PaO}_{2}\right)$. Arterial oxygen tension of grafted lung in FK (dotted lines) and PV + FK groups (solid lines) decreased as rejection developed. Therefore arterial oxygen tension in PV + FK group declined slower than that in FK group.

$10^{6}, 1 \times 10^{8}$, or $1 \times 10^{10}$ donor splenocytes immediately before lung transplantation. Control group dogs (6 animals) given no cells also received lung transplantation. As shown in Table I, portal venous inoculation with donor splenocytes alone did not affect the mean survival time of the grafted lung.

Subsequently, effects of portal venous inoculation with donor splenocytes on the graft survival of FK 506 -treated recipients were examined. For this purpose, three groups of dogs ( 6 animals each) received (1) lung transplantation followed by FK 506 treatment for 14 days (FK group), (2) lung transplantation preceded by portal venous inoculation with $1 \times$ $10^{8}$ donor splenocytes (PV group), or (3) lung transplantation preceded by portal venous inoculation with donor splenocytes and followed by FK 506 treatment for 14 days (PV + FK group). The control group was the same as that in the first experiment. As shown in Table II, the mean graft survival time was $7.2 \pm 2.3$ days in the control group, $21.5 \pm 2.4$ days in the FK group ( $p<0.05$ versus control group), $6.2 \pm 2.1$ days in the PV group, and $38.0 \pm 12.8$ days in the PV + FK group $(p<0.05$ versus control group, FK group, and PV group). In the PV + FK group, the graft survival after termination of FK 506 treatment was significantly longer $(p<0.05)$ than that in the FK group. The arterial oxygen tension (Fig. 1) of the grafted lung of individual recipients remained within the normal range for a longer period in the PV + FK group compared with findings in the FK group. Histologic examinations on day 21 revealed that, although massive infiltration of mononuclear cells was observed in the FK group (Fig. 2, $A$ ), such cellular reaction was minimal in the PV + FK group (Fig. 2, B). Prolonged graft survival after termination of FK 506 treatment observed in the PV + FK group was not caused by the reduced catabolism of FK 506, because plasma FK 506 concentrations were not significantly different between the FK group and the PV + FK group (Fig. 3).

To determine whether the dose of $1 \times 10^{8}$ splenocytes is optimal in causing the prolonged survival of the grafted lungs after termination of FK 506 administration, two groups of dogs (4 animals each) were given lower $\left(1 \times 10^{6}\right)$ and higher $(1 \times$ $10^{10}$ ) doses of donor splenocytes immediately before lung transplantation, and then these animals were treated with FK 506 for 14 days. Results in these groups were compared with those of the FK group and the PV + FK group in the second experiment. Although significant prolongation of the graft survival was observed when $1 \times 10^{8}$ cells were inoculated, the higher or lower dose showed no effects or only marginal effects on the graft survival time (Table III). 

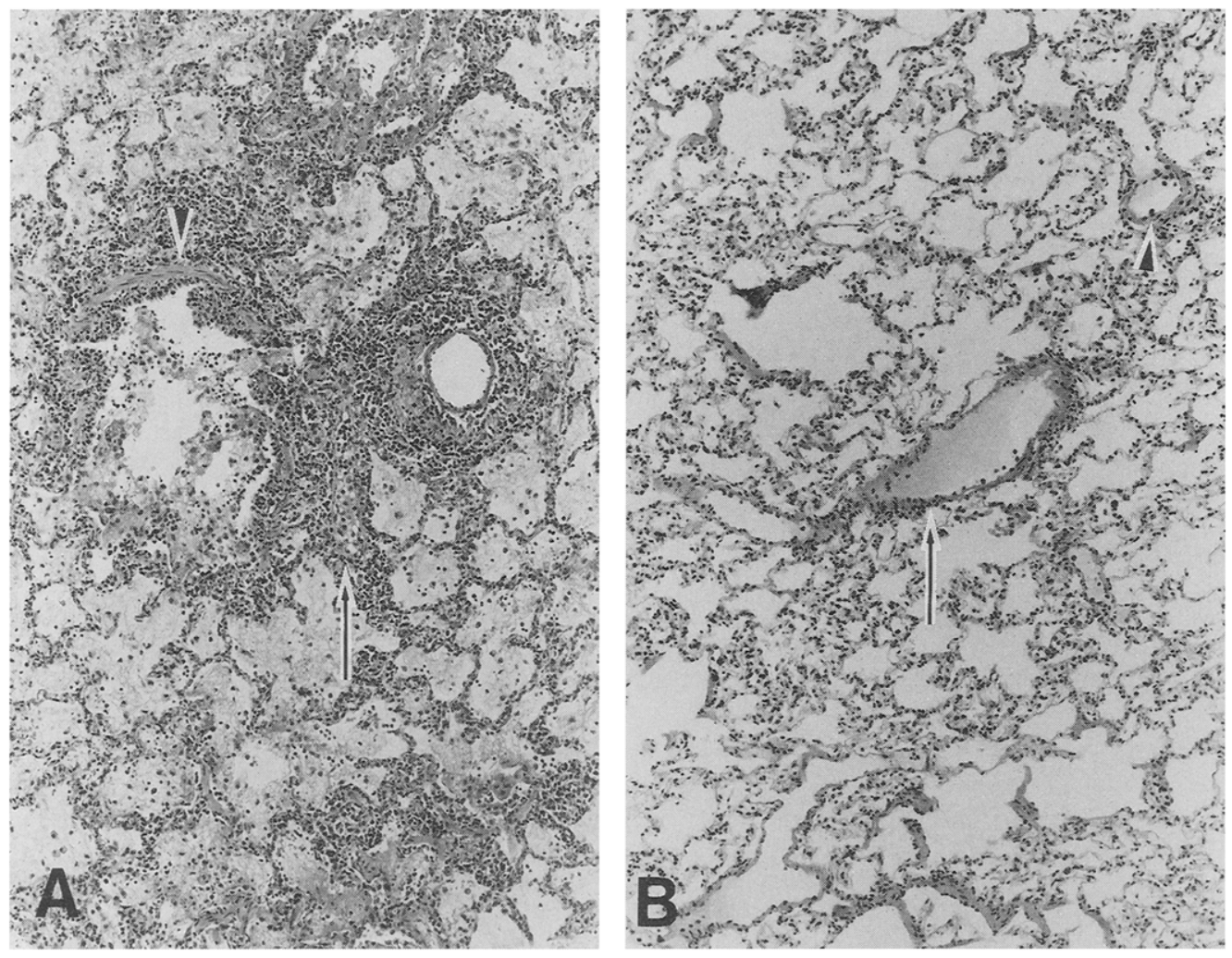

Fig. 2. Histologic studies of transplanted lung of dog from PV + FK group and FK group (hematoxylin and eosin stain; original magnification $\times 85$ ). $\mathbf{A}$, On day 21 in FK group, marked infiltration of mononuclear cells indicating moderate rejection was observed in perivascular (arrow) and peribronchial (arrowhead) areas and also in alveolar walls (grade 3; moderate rejection). B, Biopsy specimen obtained from PV + FK group on same day, on the other hand, showed minimum mononuclear leukocytic infiltration in perivascular (arrow) and peribronchial (arrowhead) areas (grade 1; minimal rejection).

\section{Discussion}

The present results show that portal venous inoculation with donor splenocytes alone did not cause prolonged graft survival in lung transplantation in dogs. However, survival time of the transplanted lung after termination of FK 506 administration was significantly prolonged by portal venous inoculation with donor splenocytes. Since Triger, Cynamon, and Wright ${ }^{18}$ first reported a reduced immune response after injections of antigen into the portal system, portal venous inoculation with donor splenocytes has been proved as the effective way to induce allograft tolerance in inbred rats. ${ }^{6-12,19,20}$ Because the efficacy of portal venous inoculation with donor splenocytes was influenced by the strain combinations, ${ }^{21}$ applicability of this effect to clinical organ transplantation should be examined in outbred combinations of large animals. In this regard, prolonged survival of kidney allografts by portal venous inocula- tion with donor splenocytes in dogs was recently reported. ${ }^{12,13}$ It is well known that the immunogenicity to induce graft rejection reaction is extremely variable among tissues probably because of the variance in the expression of histocompatibility antigens and also in the content and the type of antigen-presenting cells. ${ }^{22}$ Therefore the effects of portal venous inoculation with donor splenocytes may also be variable depending on the grafted tissues.

In small animals such as rats, immunologic tolerance to allograft was induced effectively by portal venous inoculation with donor splenocytes 1 week before organ transplantation. ${ }^{6-11}$ In terms of clinical applicability, however, preoperative portal venous inoculation with donor splenocytes is rather difficult and practically almost impossible. Therefore we performed portal venous inoculation with donor splenocytes at the time of lung transplantation. Similar to the method in our experiments, portal 


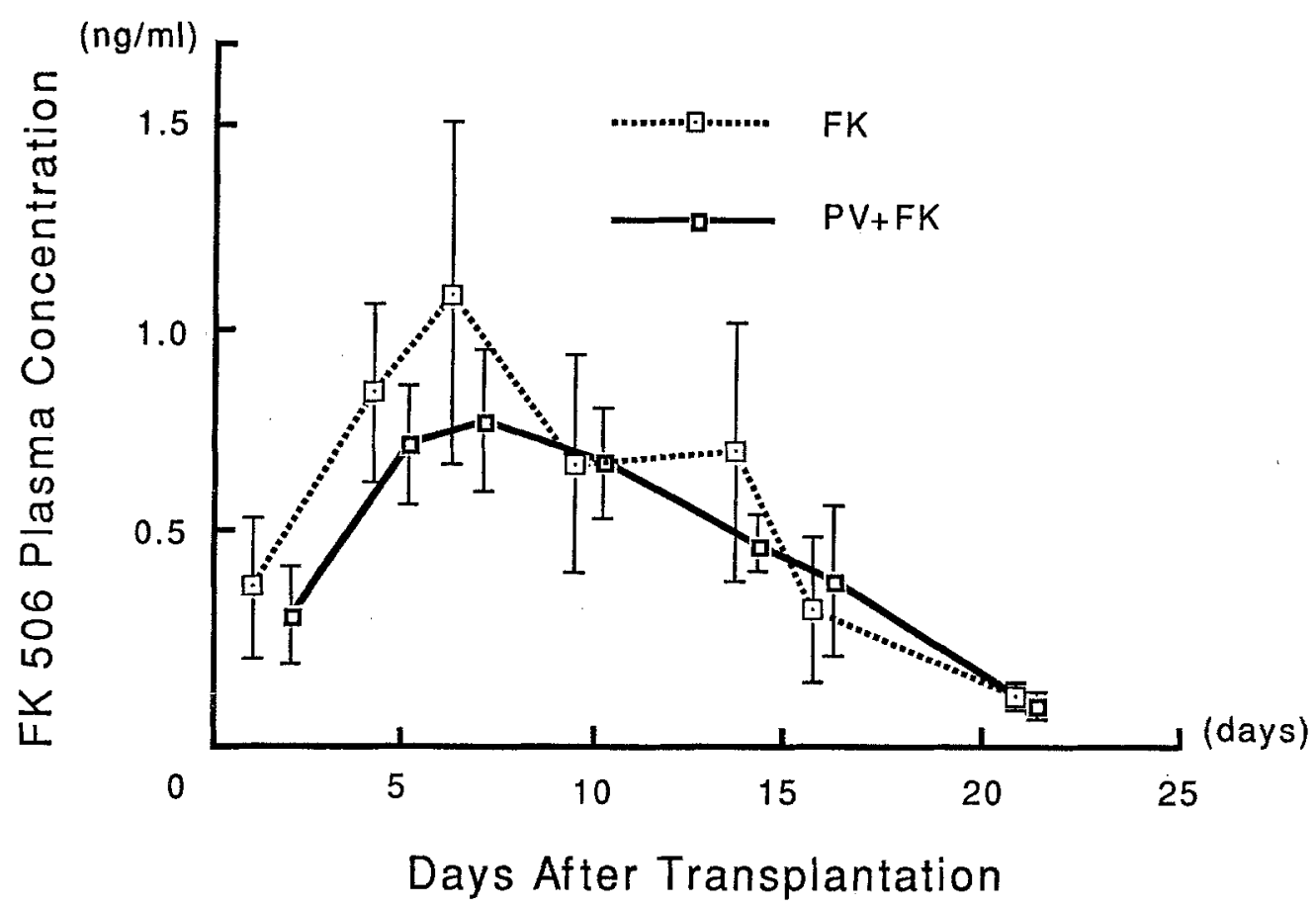

Fig. 3. Plasma trough levels of FK 506. No significant difference was observed between FK (dotted line) and PV + FK groups (solid line) throughout period examined.

Table III. Effects of portal venous inoculation with donor splenocytes on the graft survival in ling transplantation in dogs treated with FK 506

\begin{tabular}{lccl}
\hline $\begin{array}{c}\text { No. of } \\
\text { cells }\end{array}$ & $\begin{array}{c}\text { No. of } \\
\text { animals }\end{array}$ & $\begin{array}{c}\text { Graft survival } \\
\text { time (days) }\end{array}$ & Mean $\pm S D$ \\
\hline None & 6 & $18,20,21,22,23,25$ & $21.5 \pm 2.4$ \\
$1.0 \times 10^{6}$ & 4 & $23,23,26,26$ & $24.5 \pm 1.7^{*}$ \\
$1.0 \times 10^{8}$ & 6 & $26,33,34,36,36,63$ & $38.0 \pm 12.8 \dagger$ \\
$1.0 \times 10^{10}$ & 4 & $17,18,19,20$ & $18.5 \pm 1.3^{*}$ \\
\hline
\end{tabular}

All animals were treated with FK $506(0.1 \mathrm{mg} / \mathrm{kg}$ per day $)$ for 14 days starting from day of lung transplantation. Data for the groups given no cells and those given $1 \times 10^{8}$ cells appeared under FK group and PV $+\mathrm{FK}$ group, respectively, in Table II. SD, standard deviation.

*Not significantly different from control group given no cells.

$\dagger p<0.05$ versus control group given no cells.

venous inoculation with donor splenocytes was done at the time of renal transplantation in dogs. ${ }^{12,13}$ Tanigawa and associates ${ }^{23}$ reported that immunologic tolerance induced by preoperative and perioperative portal venous inoculation with donor splenocytes might be mediated by different mechanisms because mitomycin $\mathrm{C}$ treatment of donor splenocytes had different effects on graft survival.

In the present study, despite the ineffectiveness of portal venous inoculation with donor splenocytes alone on the prolongation of graft survival time, rejec- tion of the grafted lungs after termination of FK 506 administration was significantly delayed when the recipient was treated with perioperative portal venous inoculation with donor splenocytes. Because plasma FK 506 concentrations were not significantly different between the FK group and the PV + FK group, prolonged graft survival after termination of FK 506 treatment observed in the PV $+\mathrm{FK}$ group was not a result of the reduced catabolism of the drug. FK 506 is thought to suppress helper $T$ cell functions but not to inhibit suppressor $\mathrm{T}$ cell functions. ${ }^{24}$ Moreover, FK 506 was found to suppress presensitization-resistant cytotoxic T lymphocyte helpers (CD4 + CTL - Th). ${ }^{25}$ The reason the combined therapy with donor splenocyte transfer and FK 506 treatment caused longer graft survival than FK 506 therapy alone is indeed uncertain. Further study on the reactivity of $T$ cells from each group against donor antigens would elucidate underlying mechanisms.

Dose response experiments revealed that prolonged graft survival after termination of FK 506 treatment was obtained only when $1 \times 10^{8}$ splenocytes were inoculated into the portal vein. Because the higher or lower dose did not affect the graft survival time after termination of FK 506 therapy, the effective dose of donor splenocytes seems to be 
limited within a narrow range. Prolonged survival of kidney allografts in dogs by portal venous inoculation with donor splenocytes was also observed at the dose of $10^{8}$ to $10^{9} .{ }^{12,13}$ Cellular immune responses are induced at a higher or lower dose than the optimal dose for humoral immune responses. ${ }^{26}$ Therefore higher or lower doses of portal venous inoculation with donor splenocytes might induce cellular immune responses related to graft rejection.

In conclusion, although portal venous inoculation with donor splenocytes alone did not cause prolongation of the survival time of transplanted lung in dogs, the graft survival time after termination of FK 506 treatment was significantly prolonged when the recipients were treated by portal venous inoculation with donor splenocytes at the time of lung transplantation. Therefore even if portal venous inoculation with donor splenocytes alone is ineffective in the induction of allograft tolerance, this method may allow intermittent administration of FK 506 and thereby reduce the usage of the immunosuppressive agent.

We wish to thank Professors Masashi Koono, Second Department of Pathology, and Yoichi Minamishima, Department of Microbiology, Miyazaki Medical College, for their helpful suggestions. We also thank Fujisawa Pharmaceutical Co., Ltd., Osaka, Japan, for the kind donation of FK 506.

\section{REFERENCES}

1. Hardy JD, Webb WR, Dalton ML, Walker GR. Lung homotransplantation in man. JAMA 1963;186:1065-74.

2. Cooper JD, Pearson FG, Patterson GA, et al. Technique of successful lung transplantation in humans. J Thorac Cardiovasc Surg 1987;93:173-81.

3. Cooper JD. Current status of lung transplantation. Transplant Proc 1991;259:2258-62.

4. Kahan BD. Cyclosporine. N Engl J Med 1989;321:1725-38.

5. Hirai T, Wada $H$, Hasegawa $S$, et al. The immunosuppressive effect of FK 506 on canine lung transplantation. J Thorac Cardiovasc Surg 1992;103:1127-35.

6. Kenick S, Lisbona R, Marghesco D, et al. Prolonged cardiac survival following portal venous inoculation of allogeneic cells: immunologically specific entrapment of allogenic cells within the liver. Transplant Proc 1987;19:3057-60.

7. Rao VK, Burris DE, Gruel SM, et al. Evidence that donor spleen cells administered through the portal vein prolong the survival of cardiac allograft in rats. Transplantation 1988;45: 1145-6.

8. Kamei T, Callery MP, Flye MW. Pretransplant portal venous administration of donor antigen and portal venous allograft synergistically prolong rat cardiac allograft survival. Surgery 1990;108:415-22.

9. Yoshimura N, Hamashima T, Matsui S, Oka T. Prolongation of rat renal allograft survival by portal venous inoculation with donor lymphocytes. Transplant Proc 1989;21:3268-70.
10. Hamashima T, Yoshimura N, Matsui S, et al. The effects of perioperative portal venous inoculation with donor lymphocytes on renal allograft survival in the rat: II-phenotypic and functional analyses of graft-infiltrating cells. Transplantation 1990;49:171-5.

11. Nakano Y, Monden M, Valdivia LA, et al. Permanent acceptance of liver allografts by portal injection of donor spleen cells in rats. Surgery 1992;111:668-76.

12. Hirakawa K, Yoshimura N, Lee CJ, et al. Impact of perioperative portal venous administration of donor lymphocytes on survival of rat cardiac allografts and renal grafts of the mongrel dog. Transplant Proc 1993;25:346-7.

13. Tsuji T, Tashiro S, Tanabe D, et al. The effect of perioperative portal venous injection of donor spleen cells on renal allograft survival in dogs. Transplantation 1994;58:883-6.

14. Veith FJ, Richards K. Improved technic for canine lung transplantation. Ann Surg 1970;171:553-8.

15. Jones MT, Hsieh C, Yoshikawa $\mathrm{K}$, et al. A new model for assessment of lung preservation. J Thorac Cardiovasc Surg 1988;96:608-14.

16. Kobayashi M, Tamura K, Katayama N, et al. FK 506 assay past and present-characteristics of FK 506 ELISA. Transplant Proc 1991;23:2725-9.

17. Yousem SA, Berry GJ, Brunt EM, et al. A working formulation for the standardization of nomenclature in the diagnosis of heart and lung rejection: lung rejection study group. J Heart Transplant 1990;9:593-601.

18. Triger DR, Cynamon MH, Wright R. Studies on hepatic uptake of antigen: I-comparison of inferior vena cava and portal vein routes of immunization. Immunology 1973;25: 941-50.

19. Yasumura T, Kahan BD. Prolongation of rat kidney allografts by pretransplant administration of donor antigen extract or whole blood transfusion combined with a short course of cyclosporine. Transplantation 1983;36:603-9.

20. Didlake RH, Kim EK, Kahan BD. Impact of a combined regimen of cyclosporine and $3 \mathrm{M} \mathrm{KCl}$ extracted histocompatibility antigen on heterotopic rat cardiac allograft survival. Transplant Proc 1988;20:1034-7.

21. Westra AL, Petersen AH, Wildevuur ChRH, Prop J. Factors determining prolongation of rat heart allograft survival by perioperative injection of donor spleen cells. Transplantation 1991;52:606-10.

22. Welsh K, Male D. Transplantation and rejection. In: Roit I, Brosotoff J, Male D, eds. Immunology. 2nd ed. London: Churchill Livingstone, 1989:24.1-24.10.

23. Tanigawa $\mathrm{T}$, Monden $\mathrm{M}$, Nagano $\mathrm{H}$, et al. Portal tolerance in rats: effect of donor cell treatment and timing of administration. Transplant Proc 1994;26:1870-1.

24. Kino T, Hatanaka H, Hashimoto M, et al. FK 506, a novel immunosuppressant isolated from a streptomyces: I-fermentation, isolation, and physico-chemical and biological characteristics. J Antibiot (Tokyo) 1987;40:1249-65.

25. Iwata H, Kitagawa S, Sato S, et al. Suppression of allograft responses by combining donor alloantigen-specific intravenous presensitization with suboptimal doses of FK 506 . Transplantation 1993;56:173-80.

26. Parish CR, Liew FY. Immune response to chemically modified flagellin: III-enhanced cell-mediated immunity during high and low zone antibody tolerance to flagellin. J Exp Med 1972;132:298-311. 